University of Texas at El Paso

ScholarWorks@UTEP

\title{
Can We Detect Crisp Sets Based Only on the Subsethood Ordering of Fuzzy Sets? Fuzzy Sets And/Or Crisp Sets Based on Subsethood of Interval-Valued Fuzzy Sets?
}

\author{
Christian Servin \\ El Paso Community College, cservin@gmail.com \\ Gerardo Muela \\ The University of Texas at El Paso, gdmuela@miners.utep.edu \\ Vladik Kreinovich \\ The University of Texas at El Paso, vladik@utep.edu
}

Follow this and additional works at: https://scholarworks.utep.edu/cs_techrep

Part of the Computer Sciences Commons, and the Mathematics Commons

Comments:

Technical Report: UTEP-CS-17-18

\section{Recommended Citation}

Servin, Christian; Muela, Gerardo; and Kreinovich, Vladik, "Can We Detect Crisp Sets Based Only on the Subsethood Ordering of Fuzzy Sets? Fuzzy Sets And/Or Crisp Sets Based on Subsethood of IntervalValued Fuzzy Sets?" (2017). Departmental Technical Reports (CS). 1100.

https://scholarworks.utep.edu/cs_techrep/1100

This Article is brought to you for free and open access by the Computer Science at ScholarWorks@UTEP. It has been accepted for inclusion in Departmental Technical Reports (CS) by an authorized administrator of ScholarWorks@UTEP.For more information, please contact Iweber@utep.edu. 


\title{
Can We Detect Crisp Sets Based Only on the Subsethood Ordering of Fuzzy Sets? Fuzzy Sets And/Or Crisp Sets Based on Subsethood of Interval-Valued Fuzzy Sets?
}

Christian Servin, Gerardo Muela, and Vladik Kreinovich

\begin{abstract}
Fuzzy sets are naturally ordered by the subsethood relation $A \subseteq B$. If we only know which set which fuzzy set is a subset of which - and have no access to the actual values of the corresponding membership functions - can we detect which fuzzy sets are crisp? In this paper, we show that this is indeed possible. We also show that if we start with interval-valued fuzzy sets, then we can similarly detect type-1 fuzzy sets and crisp sets.
\end{abstract}

\section{Formulation of the Problem}

Fuzzy sets: a brief reminder. A fuzzy set is usually defined as a function $\mu: U \rightarrow[0,1]$ from some set $U$ (called Universe of discourse) to the interval $[0,1]$; see, e.g., $[1,2,3]$. This function is also known as a membership function.

A fuzzy set $A$ with a membership function $\mu_{A}(x)$ is called a subset of a fuzzy set $B$ with a membership function $\mu_{B}(x)$ if $\mu_{A}(x) \leq \mu_{B}(x)$ for all $x$. The subsethood relation is an order in the sense that it is reflexive $(A \subseteq A$ ), asymmetric ( $A \subseteq B$ and $B \subseteq A$ imply $A=B$ ), and transitive $(A \subseteq B$ and $B \subseteq C$ imply $A \subseteq C$ ).

Traditional (crisp) sets $S$ can be viewed as particular cases of fuzzy sets, with their characteristic functions playing the role of membership functions: $\mu_{S}(x)=1$ if $x \in S$ and $\mu_{S}(x)=0$ if $x \notin S$.

A natural question: can we detect crisp sets based only on the subsethood ordering of fuzzy sets? If we have a class $F$ of all fuzzy sets, and for each fuzzy

\section{Christian Servin}

Computer Science and Information Technology Systems Department, El Paso Community College 919 Hunter, El Paso, Texas 79915, USA, emailcservin@gmail.com

Gerardo Muela and Vladik Kreinovich

Department of Computer Science, University of Texas at El Paso, 500 W. University

El Paso, Texas 79968, USA, e-mail: gdmuela@miners.utep.edu, vladik@utep.edu 
set $A$ and for each element $x \in U$, we know the value $\mu_{A}(x)$ of the corresponding membership function, then we can easily detect which of the fuzzy sets are crisp: a fuzzy set is crisp if for every $x \in U$, we have either $\mu_{A}(x)=0$ or $\mu_{A}(x)=1$.

Suppose now that we have a class $F$ of all fuzzy sets with the subsethood ordering $A \subseteq B$ - but we have no access to the actual values of the corresponding membership functions. Based only on this ordering relation $A \subseteq B$, can we then detect crisp sets?

What if we only consider interval-valued fuzzy sets. A similar question can be asked if we consider interval-valued fuzzy sets, for which the value of the membership function is a subinterval of the interval $[0,1]: \mu(x)=[\mu(x), \bar{\mu}(x)] \subseteq[0,1]$, and $A \subseteq B$ means that $\underline{\mu}_{A}(x) \leq \underline{\mu}_{B}(x)$ and $\bar{\mu}_{A}(x) \leq \bar{\mu}_{B}(x)$ for all $x$.

What we do in this paper. In this paper, we prove that in both cases - when we consider fuzzy sets and when we consider interval-valued fuzzy sets - we can indeed detect crisp sets and type-1 fuzzy sets based only on the subsethood relation $A \subseteq B$.

\section{What If We Consider [0, 1]-Based Fuzzy Sets}

Our plan. To describe crisp sets in terms of the subsethood relation $A \subseteq B$, we will follow the following four steps:

- first, we will prove that the empty set $\emptyset$ can be uniquely determined based on the subsethood relation;

- second, we will show that 1 -element crisp sets, i.e., sets of the type $\left\{x_{0}\right\}$, can be thus determined,

- third, we will prove that 1-element fuzzy sets, i.e., fuzzy sets $A$ for which for some $x_{0} \in U$, we have $\mu_{A}\left(x_{0}\right)>0$ and $\mu_{A}(x)=0$ for all $x \neq x_{0}$, can be determined based on the subsethood relation, and

- finally, we prove that crisp sets can be uniquely determined based on the subsethood relation.

First step: how to detect an empty set? An empty set $\emptyset$ is a fuzzy set for which $\mu_{\emptyset}(x)=0$ for all $x \in U$. The detection of an empty set can be made based on the following simple result:

Proposition 1. A fuzzy set $A$ is an empty set if and only if $A \subseteq B$ for all fuzzy sets $B$.

Proof.

$1^{\circ}$. Let us first prove that when $A=\emptyset$, then $A \subseteq B$ for all fuzzy sets $B$.

Indeed, for every fuzzy set $B$, we have $0 \leq \mu_{B}(x)$ for all $x$ and thus, $\mu_{\emptyset}(x)=0 \leq$ $\mu_{B}(x)$ for all $x$, i.e., we indeed have $\emptyset \subseteq B$.

$2^{\circ}$. Let us now prove that, vice versa, if for some fuzzy set $A$, we have $A \subseteq B$ for every possible fuzzy set $B$, then $A=\emptyset$. 
Indeed, in particular, the property $A \subseteq B$ is true for the case when $B$ is the empty set. In this case, from the fact that $\mu_{A}(x) \leq \mu_{B}(x)=\mu_{\emptyset}(x)=0$, we conclude that $\mu_{A}(x)=0$ for all $x$, i.e., that $A$ is indeed the empty set.

The proposition is proven.

Second step: how to detect 1-element crisp sets based on the subsethood relation. Let us prove the following auxiliary result.

Proposition 2. A non-empty fuzzy set A is a one-element crisp set if and only if the following two conditions are satisfied:

- the class $\{B: B \subseteq A\}$ is linearly ordered and

- for no proper superset $A^{\prime}$ of $A$, the class $\left\{B: B \subseteq A^{\prime}\right\}$ is linearly ordered.

Proof.

$1^{\circ}$. Let us first prove that every 1 -element crisp set, i.e., every set of the type $A=$ $\left\{x_{0}\right\}$, satisfies the above two properties.

$1.1^{\circ}$. Let us prove the first property: that the class $\{B: B \subseteq A\}$ is linearly ordered. Indeed, for the given set $A$, we have $\mu_{A}\left(x_{0}\right)=1$ and $\mu_{A}(x)=0$ for all $x \neq x_{0}$. So, if $B \subseteq A$, i.e., if $\mu_{B}(x) \leq \mu_{A}(x)$ for all $x$, this means that $\mu_{B}(x)=0$ for all $x \neq x_{0}$. Thus, for such sets $B$, the only non-zero value of the membership function may be attained when $x=x_{0}$.

So, if we have two sets $B \subseteq A$ and $B^{\prime} \subseteq A$, then for these two sets, $\mu_{B}(x)=$ $\mu_{B^{\prime}}(x)=0$ for all $x \neq x_{0}$. Thus:

- if $\mu_{B}\left(x_{0}\right) \leq \mu_{B^{\prime}}\left(x_{0}\right)$, then, as one can easily check, we have $\mu_{B}(x) \leq \mu_{B^{\prime}}(x)$ for all $x$, i.e. we have $B \subseteq B^{\prime}$, and

- if $\mu_{B^{\prime}}\left(x_{0}\right) \leq \mu_{B}\left(x_{0}\right)$, then, as one can easily check, we have $\mu_{B^{\prime}}(x) \leq \mu_{B}(x)$ for all $x$, we have $B^{\prime} \subseteq B$.

Thus, for every two fuzzy sets $B$ and $B^{\prime}$ from the class $\{B: B \subseteq A\}$, we have either $B \subseteq B^{\prime}$ or $B^{\prime} \subseteq B$. So, this class is indeed linearly ordered.

$1.2^{\circ}$. Let us now prove that no proper superset $A^{\prime}$ of the 1-element set $A=\left\{x_{0}\right\}$ has the property that the class $\left\{B: B \subseteq A^{\prime}\right\}$ is linearly ordered.

For the set $A=\left\{x_{0}\right\}$, we have $\mu_{A}\left(x_{0}\right)=1$ and $\mu_{A}(x)=0$ for all other $x$. If $A^{\prime}$ is a superset of $A$, this means that $\mu_{A^{\prime}}(x)=1$. The fact that $A^{\prime}$ is a proper superset means that $A^{\prime} \neq A$, thus we have $\mu_{A^{\prime}}\left(x^{\prime}\right)>0$ for some $x^{\prime} \neq x_{0}$. In this case, we can define the following fuzzy set $B: \mu_{B}\left(x^{\prime}\right)=\mu_{A^{\prime}}\left(x^{\prime}\right)$ and $\mu_{B}(x)=0$ for all $x \neq x_{0}$. Then, we have $B \subseteq A^{\prime}, A \subseteq A^{\prime}$, but $B \nsubseteq A$ (since $\mu_{B}\left(x^{\prime}\right)>0$ and thus, $\mu_{B}\left(x^{\prime}\right) \not \leq \mu_{A}\left(x^{\prime}\right)=0$ ) and $A \nsubseteq B$ (since $1=\mu_{A}\left(x_{0}\right) \not \leq \mu_{B}\left(x_{0}\right)=0$ ). Thus, the class $\left\{B: B \subseteq A^{\prime}\right\}$ is indeed not linearly ordered.

$2^{\circ}$. Let us prove that, vice versa, if a fuzzy set $A$ has the above two properties, then it is a one-element crisp set.

$2.1^{\circ}$. Let us first prove, by contradiction, that we can only have one element $x$ for which $\mu_{A}(x)>0$. Indeed. if $\mu_{A}\left(x_{1}\right)>0$ and $\mu_{A}\left(x_{2}\right)>0$ for some $x_{1} \neq x_{2}$, then we can take the following fuzzy sets $B_{1}$ and $B_{2}$ : 
- $\mu_{B_{1}}\left(x_{1}\right)=\mu_{A}\left(x_{1}\right)$ and $\mu_{B_{1}}(x)=0$ for all other $x$, and

- $\mu_{B_{2}}\left(x_{2}\right)=\mu_{A}\left(x_{2}\right)$ and $\mu_{B_{2}}(x)=0$ for all other $x$.

Here, $B_{1} \subseteq A$ and $B_{2} \subseteq A$, but $B_{2} \nsubseteq B_{2}$ and $B_{2} \nsubseteq B_{1}$ - which contradicts to our assumption that the class $\{B: B \subseteq A\}$ is linearly ordered.

2.2 $2^{\circ}$ Due to Part 2.1, we have $\mu_{A}\left(x_{0}\right)>0$ for at most one element $x_{0}$; for all $x \neq x_{0}$, we have $\mu_{A}(x)=0$. Let us prove, by contradiction, that $\mu_{A}\left(x_{0}\right)=1$, i.e., that $A$ is indeed a one-element crisp set.

Indeed, if $\mu_{A}\left(x_{0}\right)<1$, then we can consider the following proper superset $A^{\prime} \supseteq A$ : $\mu_{A^{\prime}}\left(x_{0}\right)=\left(1+\mu_{A}\left(x_{0}\right)\right) / 2<1$ and $\mu_{A}^{\prime}(x)=0$ for all other $x$. Similarly to Part 1.1 of this proof, we can prove that for this superset $A^{\prime}$, the class $\left\{B: B \subseteq A^{\prime}\right\}$ is linearly ordered - which contradicts to our assumption that such a proper superset does not exist.

The proposition is proven.

Third step: how to detect 1-element fuzzy sets based on the subsethood relation. We say that a fuzzy set is a 1-element set if for some $x_{0} \in X$, we have $\mu_{A}\left(x_{0}\right)>0$ and $\mu_{A}(x)=0$ for all $x \neq x_{0}$. Let us prove the following auxiliary result.

Proposition 3. A non-empty fuzzy set $A$ is a one-element fuzzy set if and only if the class $\{B: B \subseteq A\}$ is linearly ordered.

Proof.

$1^{\circ}$. Arguments similar to Part 1.1 of the proof of Proposition 2 show that if $A$ is a one-element fuzzy set, then the class $\{B: B \subseteq A\}$ is linearly ordered.

$2^{\circ}$. Vice versa, if $A$ is not an empty set and not a one-element fuzzy set, this means that there exist at least two values $x_{1} \neq x_{2}$ for which $\mu_{A}\left(x_{1}\right)>0$ and $\mu_{A}\left(x_{2}\right)>0$. We can then take the following fuzzy sets $B_{1}$ and $B_{2}$ :

- $\mu_{B_{1}}\left(x_{1}\right)=\mu_{A}\left(x_{1}\right)$ and $\mu_{B_{1}}(x)=0$ for all $x \neq x_{1}$, and

- $\mu_{B_{2}}\left(x_{2}\right)=\mu_{A}\left(x_{2}\right)$ and $\mu_{B_{2}}(x)=0$ for all $x \neq x_{2}$.

Then $B_{1} \subseteq A$ and $B_{2} \subseteq A$, but $B_{1} \nsubseteq B_{2}$ and $B_{2} \nsubseteq B_{1}$. Thus, the class $\{B: B \subseteq A\}$ is not linearly ordered.

The proposition is proven.

Final result: how to detect crisp sets based on the subsethood relation. Let us prove the following auxiliary result.

Theorem 1. A fuzzy set $A$ is crisp if and only if every one-element fuzzy subset $B \subseteq A$ can be embedded in a one-element crisp subset of $A$.

Comment. In other words,

$A$ is crisp $\Leftrightarrow \forall A(B$ is a one-element fuzzy subset of $A \Rightarrow$

$\exists C((B \subseteq C \subseteq A) \&(C$ is a 1 -element crisp set $)))$. 


\section{Proof.}

$1^{\circ}$. Let $A$ be a crisp set, and let $B \subseteq A$ be a 1-element fuzzy set. By definition, this means that for some $x_{0}$, we have $\mu_{B}\left(x_{0}\right)>0$ and $\mu_{B}(x)=0$ for all other $x$.

Since the set $A$ is crisp, the only possible values of $\mu_{A}\left(x_{0}\right)$ are 0 and 1 . From $\mu_{B}\left(x_{0}\right) \leq \mu_{A}\left(x_{0}\right)$, we conclude that $\mu_{A}\left(x_{0}\right)>0$ and thus, that $\mu_{A}\left(x_{0}\right)=1$. So, $x_{0} \in A$ and hence $B \subseteq\left\{x_{0}\right\} \subseteq A$.

$2^{\circ}$. Vice versa, if $A$ is not a crisp set, this means that for some element $x_{0}$, we have $0<\mu_{A}\left(x_{0}\right)<1$. In this case, we can take the following 1-element fuzzy set $B \subseteq A$ : $\mu_{B}\left(x_{0}\right)=\mu_{A}\left(x_{0}\right)$ and $\mu_{B}(x)=0$ for all $x \neq x_{0}$. Here, $B \subseteq A$, but the only 1-element crisp set $C$ containing $B$ is the set $C=\left\{x_{0}\right\}$, and this 1-element crisp set is not a subset of the original set $A$ : $C \nsubseteq A$.

The theorem is proven.

\section{What If We Consider Interval-Valued Fuzzy Sets}

First step: how to detect an empty set. An empty set $\emptyset$ is an interval-valued fuzzy set for which $\mu_{\emptyset}(x)=[0,0]$ for all $x \in U$. The detection of an empty set can be made based on the following result:

Proposition 4. An interval-valued fuzzy set $A$ is an empty set if and only if $A \subseteq B$ for all interval-valued fuzzy sets $B$.

Proof is similar to proof of Proposition 1.

Second step: how to detect special 1-element interval-valued fuzzy sets based on the subsethood relation. Let's introduce an auxiliary notion. We say that an interval-valued fuzzy set $A$ is special if for some element $x_{0}$, we have $\mu_{A}\left(x_{0}\right)=[0, a]$ for some number $a>0$ and $\mu_{A}(x)=0$ for all $x \neq x_{0}$.

Proposition 5. A non-empty interval-valued fuzzy set $A$ is special if and only if the class $\{B: B \subseteq A\}$ is linearly ordered.

Proof.

$1^{\circ}$. For special sets (in the sense of the above definition), the fact that the class $\{B: B \subseteq A\}$ is linearly ordered can be proven similarly to Part 1.1 of the proof of Proposition 2.

$2^{\circ}$. Let us now prove that, vice versa, if for some non-empty interval-valued fuzzy set $A$, the class $\{B: B \subseteq A\}$ is linearly ordered, then the set $A$ is special.

$2.1^{\circ}$. Since $A$ is non-empty, there exists an element $x_{0}$ for which $\mu_{A}\left(x_{0}\right) \neq[0,0]$. Let us prove, by contradiction, that for every other element $x \neq x_{0}$, we have $\mu_{A}(x)=$ $[0,0]$.

Indeed, if we had $\mu_{A}\left(x_{1}\right) \neq[0,0]$ for some $x_{1} \neq x_{0}$, then we would be able to take the following two sets $B_{0}$ and $B_{1}$ :

- $\mu_{B_{0}}\left(x_{0}\right)=\mu_{A}\left(x_{0}\right)$ and $\mu_{B_{0}}(x)=[0,0]$ for all $x \neq x_{0}$, and 
- $\mu_{B_{1}}\left(x_{1}\right)=\mu_{A}\left(x_{1}\right)$ and $\mu_{B_{1}}(x)=[0,0]$ for all $x \neq x_{1}$.

In this case, $B_{0} \subseteq A$ and $B_{1} \subseteq A$, but $B_{0} \nsubseteq B_{1}$ and $B_{1} \nsubseteq B_{0}$. This contradicts our assumption that the class $\{B: B \subseteq A\}$ is linearly ordered.

$2.2^{\circ}$. To complete the proof of the proposition, we need to prove that the value $\mu_{A}\left(x_{0}\right)=\left[\underline{\mu}_{A}\left(x_{0}\right), \bar{\mu}_{A}\left(x_{0}\right)\right]$ has the form $[0, a]$ for some $a>0$, i.e., that $\underline{\mu}_{A}\left(x_{0}\right)=0$.

We will prove it by contradiction. Suppose that, vice versa, $\underline{\mu}_{A}\left(x_{0}\right)>0$. In this case, we can take the following sets $B_{1}$ and $B_{2}$ :

- $\mu_{B_{1}}\left(x_{0}\right)=\left[0.5 \cdot \underline{\mu}_{A}\left(x_{0}\right), 0.5 \cdot \underline{\mu}_{A}\left(x_{0}\right)\right]$ and $\mu_{B_{1}}(x)=0$ for all $x \neq x_{0}$, and

- $\mu_{B_{2}}\left(x_{0}\right)=\left[0, \underline{\mu}_{A}\left(x_{0}\right)\right]$ and $\mu_{B_{2}}(x)=0$ for all $x \neq x_{0}$.

Then, $B_{1} \subseteq A$ and $B_{2} \subseteq A$, but $B_{1} \nsubseteq \subseteq B_{2}$ and $B_{2} \nsubseteq \subseteq B_{1}$. This contradicts our assumption that the class $\{B: B \subseteq A\}$ is linearly ordered.

The proposition is proven.

Third step: how to detect 1-element type-1 fuzzy sets based on the subsethood relation. We say that an interval-valued fuzzy set is a 1-element type- 1 fuzzy set if there exists an element $x_{0}$ for which $\mu_{A}\left(x_{0}\right)=[a, a]$ for some $a>0$ and $\mu_{A}(x)=[0,0]$ for all $x \neq x_{0}$.

Proposition 6. A non-empty interval-valued fuzzy set A is a 1-element type-1 set if and only if it is satisfies the following three properties:

- the set $A$ is not special (in the sense of the above definition),

- there exists a special set $B \subseteq A$ for which the class $\{C: B \subseteq C \subseteq A\}$ is linearly ordered, and

- for no proper superset $A^{\prime}$ of $A$, the class $\{C: B \subseteq C \subseteq A\}$ is linearly ordered.

Proof is similar to the proof of Proposition 2.

Final result. Since we have subsethood, we also have union: the union of $A_{\alpha}$ is the $\subseteq$-smallest set that contains all $A-\alpha$. We can thus define type- 1 fuzzy sets as unions of 1-element type-1 fuzzy sets. Once we can detect type-1 fuzzy sets, we can use techniques from the previous section to detect crisp sets. Thus, we can indeed detect type-1 fuzzy sets and crisp sets based only on subsethood relation between interval-valued fuzzy sets.

\section{References}

1. G. Klir and B. Yuan, Fuzzy Sets and Fuzzy Logic, Prentice Hall, Upper Saddle River, New Jersey, 1995.

2. H. T. Nguyen and E. A. Walker, A First Course in Fuzzy Logic, Chapman and Hall/CRC, Boca Raton, Florida, 2006.

3. L. A. Zadeh, "Fuzzy sets", Information and Control, 1965, Vol. 8, pp. 338-353. 